\title{
Measurement of the Complexity of Variation Points in Software Product Lines
}

\author{
Yuqing Lin, Huilin Ye and Jianmin Tang \\ School of Electrical Engineering and Computer Science \\ The University of Newcastle, NSW 2308, Australia \\ e-mail: \{yuqing.lin, huilin.ye, jianmin.tang\}@newcastle.edu.au
}

\begin{abstract}
Feature models are used in member product configuration in software product lines. A valid product configuration must satisfy two kinds of constraints, multiplicity of each variation point and dependencies among the variants in a product line. The combined impact of the two kinds of constrains on product configuration should be well understood. In this paper we propose a measurement, called VariationRank, that combines the two kinds of constraints to assess the complexity of variation points in software product lines. Based on the measurement we can identify those variation points with the highest impact on product configurations in a product line. This information could be used as guidance in product configuration as well as for feature model optimization in software product lines. A case study is presented and discussed in this paper as well.
\end{abstract}

\section{Introduction}

Software Product Line Engineering (SPLE) is gaining focuses from both industry and research groups. SPLE empowers high-level constructive software reuse by exploiting commonality and managing variability among the member products in a product family. SPLE normally includes two processes, domain engineering and application engineering. In domain engineering a domain model is developed to capture the commonalities and variability between the various resulting products in a product line. In application engineering, the product variability is exploited and refined. Individual products can then be "assembled" based on the model developed in the process of domain engineering. SPLE promotes systematic software reuse and significantly improves in time-to-market, cost, productivity, and quality of the software development.
Feature oriented approaches [2, 3, 4, 6] have been used in domain engineering in software product lines. The features identified from a product line are prominent and distinctive system requirements or characteristics that are visible to various stake holders in the product family [1]. A feature model is developed to define features and their usage constraints in a product line. An important concept in feature models is variation point. Currently existing approaches usually use a tree structure to organise features. To configure a member product of a product line using a feature tree, if a parent node of the tree has one or more direct variable child features, called variants, a decision must be made on the choice of the variants. The parent feature representing a decision point together with its possible choices (variants) is defined as variation points [2]. Feature models are used for product configuration in product lines. A member software product in the product line is defined by a unique combination of legally selected features in the product line. The process of selecting features from a feature model for a member product in a product line is called product configuration. In order to effectively configure a member product in a product line the constraints of how to select the variants at each variation point to construct a valid member product should be well understood. There are mainly two kinds of constraints contributed to product configuration in product lines. One is the multiplicity of each variation point that specifies how many variants can be selected from each variation point. Dependency among the variants in a product line is another kind of constraint. Even if a selection of a feature at a variation point satisfies the multiplicity constraint it may not satisfy the dependency constraint if the selected feature has interdependencies with other variants. Current feature oriented approaches support the representations of both kinds of constraints respectively but we think that it is important to assess the complexity of both kinds of constraints in a single combined measurement.

In this paper we propose a measurement of complexity for variation points based on the Page Ranking Algorithm [7]. The measurement takes the two kinds of constraints into 
consideration to measure how complex a variation point is. Based on the measurement we can identify those variation points with the highest impact on product configurations. The measurement can also be used to access the quality of feature models. The assessment result can be used for feature model optimization.

The reminder paper is organized as follows. Second 2 presents our approach of how to construct a graph based on a feature model and how to calculate VariationRank from the graph. A case study is given in Section 3. Section 4 specifies future work and concludes the paper.

\section{Calculation of VariationRank}

In a feature model, a multiplicity is associated with each variation point specifying how many variants can be selected from this variation point. There are 4 kinds of multiplicity $[2,6]$ :

- 1: One and only one feature can be selected used to represent alternative variants.

- 1..*: One or more features can be selected used to represent multiple alternative variants.

- 0..1: no feature or at most one feature can be selected used to represent optional alternative variants.

- $0 . . *$ : no feature or more features can be selected used to represent optional multiple alternative variants.

In addition to this type of the relationship, a variant often has dependencies on the other variants in a product line. The dependent relationships among variants have been categorized into 3 different types [4, 6]:

- require-relationship: If a variant requires, or uses, another variant to fulfil its task, there is a requirerelationship between the two variants.

- excludes-relationship: If two variants mutually exclude each other there is a bi-directional excludesrelationship between the two variants.

- impact-relationship: When the selection of one variant has impact on another variant, there is an impactrelationship between the variants.

we combine the constraints of multiplicity and dependencies into a single measurement called VariationRank to estimate the complexity of variation points, i.e how much influence (impact) the variation point has on the rest of the feature model. We will first construct a graph. Page ranking algorithm will then be applied on the graph to calculate the weight of each vertex in the graph, where the weight is VariationRank indicating the complexity of the vertex.

We take an example to give more details about how to construct a graph for a feature model. Each vertex in the graph represents a possible selection of variant(s) at a certain variation point. The selection may be a single variant or a combination of variants at a variation point. The possible selections at a certain variation point are constrained by the multiplicity and the dependent relationships among the set of variants at the variation point.
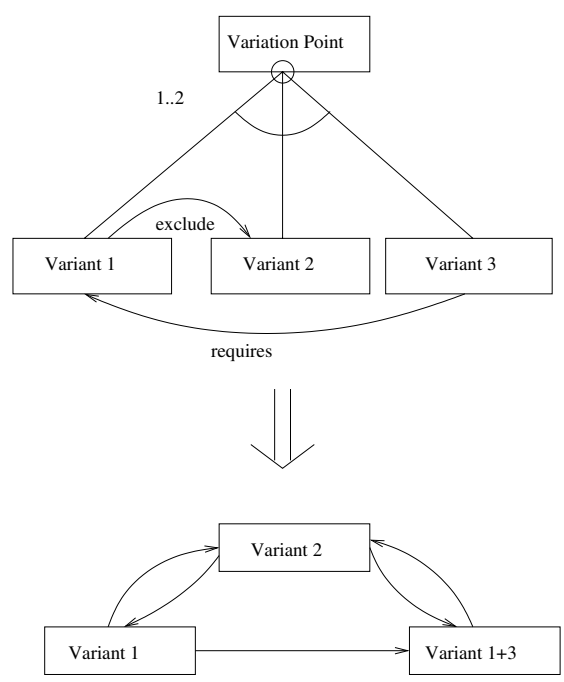

Figure 1. The transformation of multiplicity into the relationships among the variants

In the example shown in Figure 1, we have three variants 1,2 , and 3. Based on the multiplicity of $1 . .2$ the possible selections at this variation point will be $\{1,2,3,1+$ $2,1+3,2+3\}$. Considering the dependencies between the three variants as indicated in Figure 1, as Variant 1 excludes Variant $2,1+2$ is not a possible selection; as Variant 3 requires Variant 1 , Variant 3 alone and $2+3$ are also not possible selections. Thus the set of Possible Selections of Variant(s), called PSV, at this variation point VP is $P S V(V P)=\{1,2,1+3\}$. Each element in this set is a vertex in the constructed graph for this variation point. The dependent relationships for these vertices are obviously exclude-relationship as shown in Figure 1.

Once we have worked out how to construct a graph for individual variation points, these graphs can be connected by the dependencies among the variants at different variation points specified in a feature model to form a bigger directed graph for the feature model. Each vertex in the graph is a 
possible selection of variant(s) at a certain variation point. Two vertices is linked by an arc if a dependent relationship exists between the two vertices, for example, if variant $a$ requires variant $b$, then there will be an arc from $a$ to $b$. In this paper, we do not distinct differences among the relationships and treat all arcs with the same weight. Also, in our approach, we support the multiple relationships among the variants, which will be modelled as multiple arcs between two vertices in the graph.

Once we have the directed graph, it is easy to use the Page Ranking algorithm to calculate a "rank" for each vertex in the graph. The ranking algorithm is used very often in ranking the hyperlinked set of documents on the Web, and it gives a relative importance of the elements in the set. This algorithm has been used by Google and many other search engine. The ranking is basically a voting system. Each element in the system counts the number of supports from the other elements, including both the element directly associated with it as well as the elements indirectly associated. The elements with the most number of supports are the most important in the system [7].

We apply the similar idea in our case, we will calculate how much a selection of variant(s) will affect the selection of other variants. If selecting a variant $v_{i}$ in a feature model directly or indirectly restricts the selection of the other variations, then the $v_{i}$ is considered as having impact to the product configuration. The higher the impact, the more important is the variant in the model. Note here that we will have to switch the direction of the arcs in the graph we obtained in the previous step, this is to fit the "voting" system used in the page ranking algorithm. For instance, considering two variants $a$ and $b$, if $a$ requires $b$, it means that select $a$ will have impact on the selection of $b$, therefore, $b$ should vote for $a$ to indicate the importance of $a$.

To calculate the VariationRank of a variant, denote by $V R$, we will calculate iteratively as described below. For a variant or a combination of variants $v_{i}$,

$$
V R\left(v_{i}\right)=\frac{1-d}{N}+d \sum_{v_{j} \in M\left(v_{i}\right)} \frac{V R\left(v_{j}\right)}{L\left(v_{j}\right)}
$$

- $L\left(v_{j}\right)$ - each variant spreads its vote out evenly amongst all of its outgoing links. The number of outgoing links for variant or a combination of variant $v_{i}$ is $L\left(v_{i}\right)$.

- $V R\left(v_{j}\right) / L\left(v_{j}\right)$ - So if $v_{i}$ has a link from $v_{j}$, then the share of the vote page $v_{i}$ will get is $V R\left(v_{j}\right) / L\left(v_{j}\right)$.

- $d$ - The damping factor. When we consider the derived dependency, it is clear that the further away a variant $v_{j}$ is from $v_{i}$, the less influence it is to $v_{i}$. Therefore, in here, the total vote is "damped down" by multiplying it by the damping factor. It is generally assumed that the damping factor $d$ will be set around 0.85 in calculating the PageRank, so in here, we use the same value.

- $(1-d)$ - In the calculation, the $(1-d)$ at the beginning means that even if a variant has no relation to the other variants, it will still get a small VariantRank of 0.15 (i.e. $1-0.85$ ). This will make the calculation easier.

- $M\left(v_{i}\right)$ is the set of variants and combinations of variants that link to $v_{i}$.

- $N$ is the total number of variants or combination of variants.

The VariationRank of a variation point $v p_{x}$ is the average of the VariationRank of all the elements in $P S V\left(v p_{x}\right)$. The VariationRank could tell how much impact of a variation point on the product configuration in a product line. This measurement also could be used as the objective function for feature model optimization. It could help designers to identify the most complex variation points which should be redesigned or improved.

\section{A Case Study}

In this section, we will use an example to demonstrate our approach. Figure 1 shows a feature model for the Order_Process product line. This model is adapted from $[5,6]$. The variation points, their multiplicity, and the dependencies between the variants are clearly presented in the figure. Based on the method specified in Section 2 we identify the following PSV sets for the variation points in Figure 2. To make it simple, when we cite a variation point we will use the feature name of the parent node to represent the variation point. Please note Shipment is the parent of two different variation points. We use Shipment_S to represent Shipment and its variant Shipping, use Shipment_P to represent Shipment and its variants, Packing_Slip and Package_Tracking.

- $\operatorname{PSV}($ Fulfillment $)=\{$ Service_Delivery, Shipment, Electronic_Delivery\}.

- $\operatorname{PSV}($ Transaction $)=\{$ Shipping_Cost $\}$.

- $\operatorname{PSV}($ Invoice $)=\{$ Printed_Invoice, $\quad$ On_line_Display, Printed_Invoice + On_line_Display $\}$.

- $\operatorname{PSV}($ Payment $)=\{$ Pay_By_Bill, Credit_Card, Pay_On_Delivery, Frequent_Flyer\}.

- $\operatorname{PSV}($ Shipment_S $)=\{$ Shipping $\}$. 
- PSV(Shipment_P $)=\{$ Packing_Slip, Package_Tracking, Packing_Slip + Package_Tracking $\}$.

For easy presentation, we labelled all the elements identified in the above PSV sets with a number (see Table 1). Based on these elements and the dependent relationships among the elements we construct a graph (shown in Figure 3) to calculate VariationRank for each vertex and each variation point. Our experimental results are listed in Table 2 and 3, and the largest value of VariationRank is written in bold.

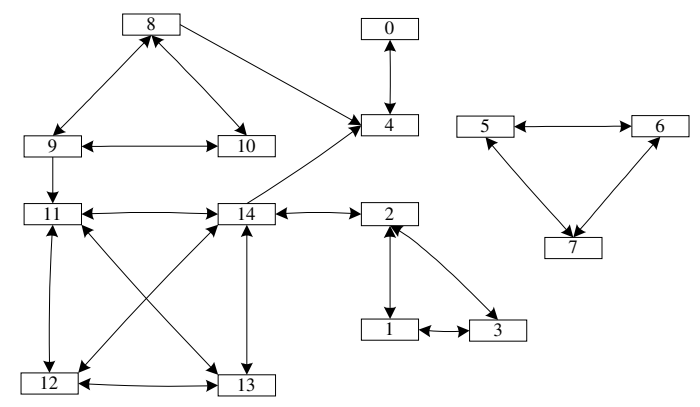

Figure 3. A graph derived from the feature model shown in Figure 2

\begin{tabular}{|c|c|}
\hline Label & Variant or Combined Variants \\
\hline 0 & Shipping_Cost \\
\hline 1 & Service_Delivery \\
\hline 2 & Electronic_Delivery \\
\hline 3 & Shipment \\
\hline 4 & Shipping \\
\hline 5 & Packing_Slip \\
\hline 6 & Package_Tracking_No \\
\hline 7 & Packing_Slip + Package_Tracking_No \\
\hline 8 & Printed Invoice \\
\hline 9 & On_line_Display \\
\hline 10 & Printed Invoice + On_cline_Display \\
\hline 11 & Pay_By_Bill \\
\hline 12 & Credit_Card \\
\hline 13 & Pay_On_Delivery \\
\hline 14 & Frequent_Flyer \\
\hline
\end{tabular}

Table 1. The label of the variants or combination of variants

From the results of the computation,

1) We found that the variant "Shipping" has the highest VariationRank. This means selecting this variant will have the highest impact on the selection of other variants in the product line. With this simple example, this can be observable from the feature model. However, if it is a large and

\begin{tabular}{|c|c|c|c|}
\hline Number & PR(Number) & Number & PR(Number) \\
\hline 0 & 0.1249 & 8 & 0.0313 \\
\hline 1 & 0.0519 & 9 & 0.0313 \\
\hline 2 & 0.0689 & 10 & 0.0281 \\
\hline 3 & 0.0519 & 11 & 0.0691 \\
\hline 4 & $\mathbf{0 . 1 3 3 6}$ & 12 & 0.0621 \\
\hline 5 & 0.0667 & 13 & 0.0621 \\
\hline 6 & 0.0667 & 14 & 0.0850 \\
\hline 7 & 0.0667 & & \\
\hline
\end{tabular}

Table 2. The VariationRank of each vertex in Figure 3

\begin{tabular}{|c|c|}
\hline Variation Points & PR(Variation Points) \\
\hline Transaction & 0.1249 \\
\hline Fulfillment & 0.0576 \\
\hline Shipment_S & $\mathbf{0 . 1 3 3 6}$ \\
\hline Shipment_P & 0.0667 \\
\hline Invoice & 0.0302 \\
\hline Payment & 0.0696 \\
\hline
\end{tabular}

Table 3. The VariationRank of all the variation points in Order-Process

complex feature model it is difficult to identify those variants with highest impact on product configuration through direct inspection of the feature model. Thus the VariationRank provides a good means to identify such high impact variants.

2) We also found the ranking of variation points in this product line. The variation point "Shipment_S" has the highest VariationRank as its variant "Shipping" depends heavily on the other variants in the product line. The variation point "Invoice" has the lowest VariationRank as its variants "Printed Invoice" and "on_line Display" do not depend on other variants in the product line but only are depended by some of the other variants, i.e., selecting variants at this variation point will not have much impact on the selections of other variants.

3) The measurement of VariationRank can be used for product configuration and feature model optimization. In the configuration process we should consider the most complex variation points first. In this way the configuration may be more efficient as we can avoid some of the corrections of the mistakes made in the earlier steps of the configuration. A high VariationRank of a variation point may also suggest that the variation point may not be abstracted at an appropriate level and further splitting of the variation point may 


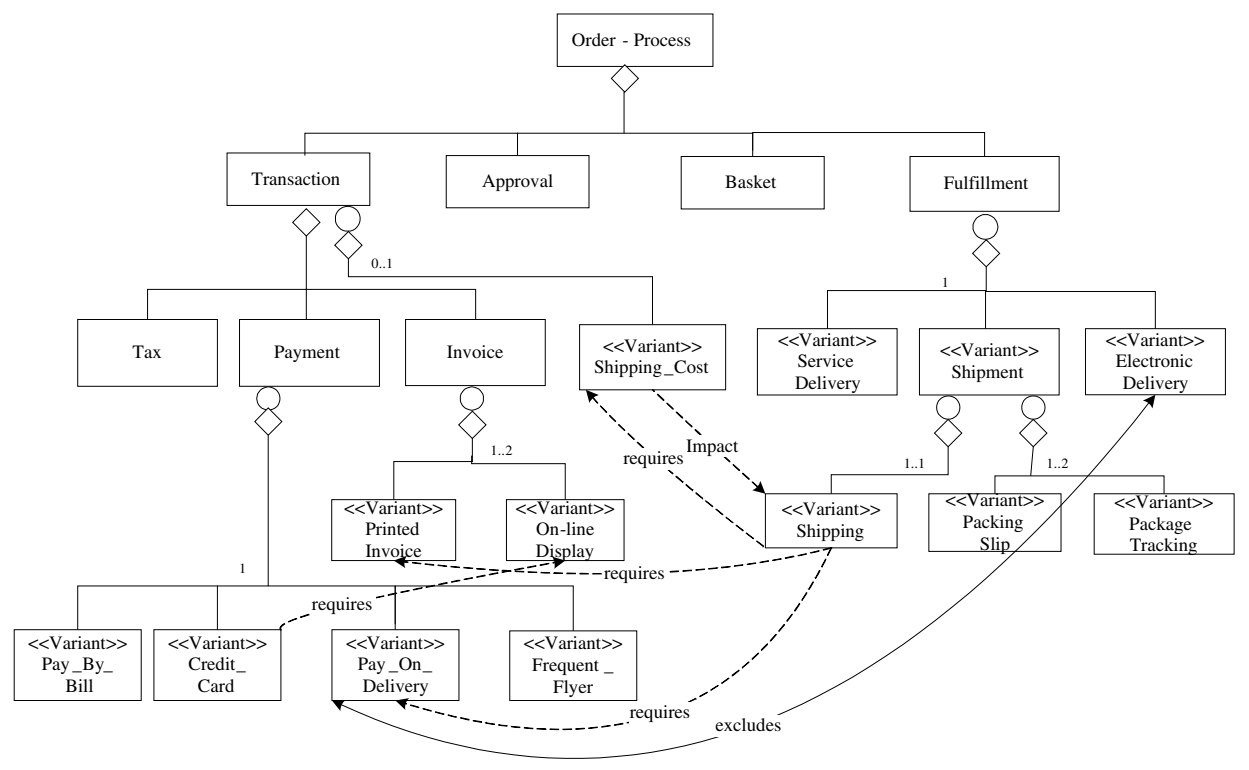

Figure 2. A feature model for Order-Process software product line

be possible to reduce the value of VariationRank to optimise the feature model.

\section{Conclusion and Future Work}

In this paper, we proposed a measurement, called VariationRank, to estimate the complexity of variants and variation points in feature models for software product lines. This measurement has been applied in a case study, a feature model for the Order_Process product line. The results of the VariationRanks are consistent with our direct observations of the feature model and these results can be used in product configuration and feature model optimisation.

In this approach, we have not considered the differences among the different types of the dependent relationships. If we could define the relationships more precisely then we could use weighted hyper-graphs to present the relationships of the variation points in future. Certainly, there will be more information revealed from the ranking results.

\section{References}

[1] K. Lee, K. Kang and J. Lee, Concepts and Guidelines of Feature Modelling for Product Line Software Engineer- ing. Proc. of 7th Int. Conf. of Software Reuse, LNCS, 2319 (2002), 62-67.

[2] M. Riebisch, K. Böllert, D. Streitferdt and I. Philippow, Extending Feature Diagrams with UML Multiplicities. Proc. of the Sixth Conf. on Integrated Design and Process Technology (2002).

[3] K. Kang, K. Lee, J. Lee and S. Kim, Feature-Oriented Product Line Software Engineering: Principles and Guidelines, to be published in Domain Oriented Systems Development Practices and Perspectives, Gordon Breach Science Publishers, UK, (2002).

[4] Kang, K., et al. Feature-Oriented Domain Analysis (FODA) Feasibility Study (CMU/SEI-90-TR-21, ADA 235785). Pittsburgh, PA: Software Engineering Institute, Carnegie Mellon University, (1990).

[5] M. Clau $\beta$, Modelling Variability with UML. In GCSE 2001 Young Researchers Workshop, Sept. (2001).

[6] H. Ye and H. Liu, An Approach to Modelling Feature Variability and Dependencies in Software Product Lines, IEE Proceedings - Software, 152 (2005), 101109.

[7] S. Brin and L. Page, The anatomy of a large-scale hypertextual Web search engine, Proceedings of the seventh international conference on World Wide Web 7:(1998), 07-117. 